\title{
MEJORA DE LAS CARACTERÍSTICAS MECÁNICAS DE HORMIGONES CON INCLUSIÓN DE DOS TIPOS DE CENIZAS: LODO DE DEPURADORA Y CÁSCARA DE ARROZ. UNA ALTERNATIVA SOSTENIBLE CON AHORRO DE MATERIAS PRIMAS Y REDUCCIÓN DE RESIDUOS
}

\author{
F. BAEZA-BROTONS ${ }^{1}$, P. GARCÉS ${ }^{1}$, J. PAYÁ ${ }^{2}$, O. GALAO $^{1}$, M. GARCÍA-ALBERTI ${ }^{3}$ \\ ${ }^{1}$ Departamento de Ingeniería Civil, Universidad de Alicante, Carretera de San Vicente del Raspeig S/N, \\ 03690 San Vicente del Raspeig, Alicante, España. \\ 2 Instituto de Ciencia y Tecnología del Hormigón (ICITECH), Universitat Politècnica de València, \\ Camino de Vera S/N, 46022 València, España. \\ 3 E.T.S. de Ingenieros de Caminos, Canales y Puertos, Universidad Politécnica de Madrid, \\ C/Profesor Aranguren s/n, 28040 Madrid, España.
}

\begin{abstract}
RESUMEN
Se evalúa la efectividad de incorporar dos residuos en matrices cuyo conglomerante es cemento Portland: ceniza de lodo de depuradora de aguas residuales (CLD) y ceniza de cáscara de arroz (CCA). Se plantean seis dosificaciones: hormigón patrón; adición con respecto al cemento del 10\%CCA; sustitución del 30\% del cemento por $20 \%$ CLD+10\%CCA; la anterior con adición 10\%CCA; sustitución del $40 \%$ del cemento por $20 \%$ CLD+20\%CCA; y la anterior con adición 10\%CCA. La resistencia a compresión de varias dosificaciones supera la clase resistente del cemento y la del patrón, destacando la sustitución equivalente del cemento cercana al 50\%. Esto se debe a un doble efecto de las cenizas: la matriz es más compacta por aporte de material fino y su carácter puzolánico.
\end{abstract}

Palabras clave: hormigón; resistencia mecánica; valorización residuos.

\begin{abstract}
The effectiveness of incorporating two residues in matrices whose conglomerate is Portland cement is evaluated: sewage sludge ash from sewage treatment plant (CLD) and rice husk ash (CCA). There are six dosages: control mix; addition of $10 \%$ CCA respect to cement; replacement of $30 \%$ of cement by $20 \%$ CLD + $10 \%$ CCA; the previous one with $10 \%$ CCA addition; replacement of $40 \%$ of cement by $20 \%$ CLD $+20 \%$ CCA; and the previous one with $10 \%$ CCA addition. The compressive strength of several concretes exceeds the resi stant class of cement and control mix, highlighting the equivalent substitution of cement close to $50 \%$. This behaviour is attributed to a double effect of the ashes: the matrix is more compacted due to the contribution of fine material and its pozzolanic character.
\end{abstract}

Keywords: concrete; mechanical strength; waste valorization.

\section{RESUMO}

Neste trabalho é avaliada a eficácia da incorporação de dois resíduos em matrizes a base de cimento Portland: cinza de lama da estação de tratamento de esgoto (CLD) e cinza de casca de arroz (CCA). Serão estudadas seis dosagens: traço controle; traço com adição de $10 \%$ de CCA em relação ao cimento; traço com substituição de $30 \%$ de cimento por $20 \%$ de CLD + 10\% de CCA; traço similar ao anterior porém com adição de $10 \%$ de CCA; traço com substituição de $40 \%$ de cimento por $20 \%$ de CLD + 20\% de CCA; e traço similar ao anterior porém com $10 \%$ de adição de CCA. A resistência à compressão de várias dosagens estudadas supera a classe resistente do cimento Portland e a também a resistência do traço controle, destacando que a substituição equivalente do cimento é próxima a $50 \%$. Isto pode ser devido a um efeito duplo das cinzas: redução de vazios devido à presença de material fino e também devido ao caráter pozolânico do material.

Palavras-chave: concreto; resistência mecânica; valorização de resíduos. 


\section{INTRODUCCIÓN}

Para mantener el equilibrio entre el desarrollo tecnológico y el medio ambiente se acuñó, en 1987, el término "desarrollo sostenible". Dicho término fue definido como: forma de progreso que satisface las necesidades del presente, sin comprometer las necesidades de las futuras generaciones en todos los aspectos: social, humano y ambiental. A raíz de esto, se han llevado a cabo diversas convenciones con el reto de promover el desarrollo sostenible, siendo el protocolo de Kioto, firmado en 1997, el tratado más relevante (Roskovic et al, 2005).

En línea con lo anterior, el avance hacia la economía circular se refleja en la legislación nacional española - en la ley de residuos (Ley 22/11, 2011) o en el Plan Estatal Marco de Gestión de Residuos (PEMAR, 2015) - a través de la aplicación, en todos los flujos de residuos incluidos, del principio de jerarquía establecido en la normativa comunitaria. Este principio, establece que la prevención debe ser la prioridad principal en relación con la política de residuos, seguida por este orden: por la preparación para la reutilización, el reciclado, otras formas de valorización, incluida la valorización energética, siendo la eliminación de residuos, fundamentalmente a través del depósito en vertedero, la última opción de la jerarquía para gestionar los residuos.

Precisamente, la actividad de la construcción es una gran demandante de recursos y materiales, lo que la convierte en un sector con enorme potencial para el aprovechamiento de residuos, tanto de los generados por su propia actividad, como de los procedentes de otros sectores, por tanto con capacidad para desarrollarse de manera sostenible. La reutilización, reciclaje o revalorización de dichos residuos permite reducir el consumo energético, preservar las fuentes de recursos naturales no renovables, y reducir la elevada cantidad de material que, al no poder incorporarlo a los ciclos naturales o a las líneas de producción industrial por razones tecnológicas o económicas, finalmente se destina a vertedero. En este sentido, el Centro de Estudios y Experimentación de Obras Públicas, edita una monografía titulada Catálogo de Residuos Utilizables en Construcción, donde se encuentran los residuos reutilizados actualmente o que se encuentran en fase experimental (CEDEX, 2019).

En el presente trabajo se trata de evaluar la efectividad de incorporar en una matriz cuyo conglomerante es el cemento Portland, y de manera combinada, dos cenizas de residuos como la ceniza de lodo de depuradora (CLD), procedente de plantas de tratamiento de aguas residuales, y la ceniza de cáscara de arroz (CCA).

El uso experimental de las CLD incorporadas a matrices cementantes es sobradamente conocido debido a la numerosa bibliografía existente. Por ejemplo, cuando fueron utilizadas como sustitución parcial del cemento en proporciones del 10\%, o con $2 \%$ de sustitución de arena, las probetas de hormigón presentaron resistencias a la compresión similares a las muestras control. En cuanto al estudio de lixiviación, los resultados indicaron que la mezcla de cenizas con cemento y arena para producir mortero u hormigón indujeron una estabilización de Mo y Se, y de este modo constituyen en sí mismo un buen tratamiento (estabilización/solidificación) de las cenizas (Chen et al., 2013). Otros trabajos han demostrado también que morteros que contienen CLD presentan buenas propiedades mecánicas (Monzó et al., 1996; Alcocel et al., 2006). La mejora observada se debe a la actividad puzolánica de la ceniza (Payá et al., 2002; Cyr et al., 2007), aunque recientes investigaciones han revelado que, comparado con materiales puzolánicos ya conocidos, la actividad puzolánica de las cenizas es débil en el mejor de los casos (Donatello et al., 2010). En un estudio en el que se usó este residuo en hormigones destinados a prefabricación de bloques, fue diseñada una escala de adiciones de la ceniza CLD respecto al cemento $(5,10,15$ y $20 \%)$, y la sustitución de parte de la arena por este material. Se comprueba que la adición de CLD en dichos hormigones curados durante 28 días, aporta una densidad y resistencia cercana al patrón (sin adición) y hace disminuir considerablemente la absorción de agua. La sustitución 
de arena por la adición mineral hace mejorar notablemente los parámetros anteriores (Baeza-Brotons et al, 2014a).

En cuanto al uso de la CCA, en un trabajo (Rawaid, 2012) en el que se utilizó CCA como sustituto parcial del cemento en morteros y hormigones, en general, los resultados para sustituciones de cemento por $25 \% \mathrm{CCA}$, mostraron los mismos o mejores resultados en comparación con el hormigón convencional, con una tasa de ganancia de la resistencia a edades tempranas más baja en comparación con el hormigón patrón, lo que puede ser debido a la lenta velocidad de reacción de la CCA. Mayores proporciones de CCA (40\%) podrían ser usadas para hormigones no estructurales donde la resistencia no es crítica. En otro de los trabajos consultados (Rahmat et al, 2011), se estudió el efecto de la ceniza de cáscara de arroz en las propiedades del hormigón y la durabilidad. Para establecer la proporción adecuada de CCA en la sustitución parcial de cemento, se produjeron mezclas de hormigón con 0-30\% CCA y se determinaron sus propiedades mecánicas, así como el efecto de CCA en la homogeneidad del hormigón. Se evaluó la durabilidad de las muestras expuestas a ambientes agresivos (5\% de $\mathrm{NaCl}$ con ciclo seco-húmedo) durante once meses. En general, los resultados indican que la sustitución parcial del cemento por CCA ha mejorado la durabilidad y la homogeneidad, pero no aumentó la resistencia a compresión del hormigón a edad temprana. Sin embargo, los hormigones que contienen CCA mostraron mayor resistencia a la compresión en las edades más avanzadas. La exploración por microscopía electrónica de barrido sobre la microestructura de las muestras de mortero mostró que los productos de reacción puzolánica de la CCA llenan los poros y esto explica el rendimiento mecánico superior del mortero con CCA.

El número de trabajos en los que se combinan CLD con otros residuos, incluyendo CCA, es muy escaso. En uno de ellos (Baeza-Brotons et al, 2014b) se realizan combinaciones binarias y ternarias de CLD con polvo de mármol, cenizas volantes (CV) y CCA, como sustitución parcial del cemento portland en pastas y morteros. Son identificados efectos puzolánicos de las mezclas, densidades similares al patrón, y absorciones mejoradas al unir residuos. En general la resistencia a compresión alcanza o supera la clase resistente del cemento, y combinando CLD, CV y CCA (sustitución 30\%), se incrementa este parámetro un $9 \%$ con respecto al patrón. En otro artículo de los mismos autores con similares combinaciones de residuos que en el trabajo anterior, pero aplicado a hormigones (Baeza-Brotons et al, 2015), se comprueba que la sustitución de cemento por CLD supone una disminución de la densidad y de la resistencia respecto a la muestra patrón, sin embargo, las combinaciones con otros residuos mejoran notablemente las características de los materiales cementantes.

En base a todo lo anterior, el objetivo principal del presente trabajo es profundizar en el estudio del uso combinado de residuos como la CLD y la CCA en hormigones, optimizando las sinergias generadas. La importancia del trabajo radica en dos cuestiones: en primer lugar, el hecho de que estos estudios son poco numerosos y hay poca información al respecto; en segundo lugar, para llevarlo a cabo se utilizará un doble concepto aplicado de manera simultánea: sustitución de cemento (por la combinación binaria de las cenizas) y adición con respecto al cemento (de las CCA), con lo que se pretende disminuir lo máximo posible la cantidad de cemento utilizado en las mezclas y aumentar la cantidad de residuo valorizado.

\section{PROCEDIMIENTO}

Se plantean seis dosificaciones de hormigones en las que los residuos sustituyen y/o adicionan al cemento. De esta forma, además de la muestra patrón "Figura 1", son estudiadas las siguientes mezclas: adición del 10\%CCA; sustitución de 30\% del cemento por la combinación 20\%CLD+10\%CCA; la sustitución anterior más adición 10\%CCA; sustitución del 40\% del cemento por la combinación 20\%CLD+20\%CCA; y por último, la sustitución anterior más adición 10\%CCA. En la "Tabla 1" se 
detallan dichas dosificaciones con sus cantidades en $\mathrm{kg} / \mathrm{m}^{3}$, con una relación agua/bínder de 0.5 en todos los casos. Se fabrican 3 probetas por mezcla y se conservan durante 28 días en cámara climática en condiciones de humedad y temperatura constante $\left(90 \% \mathrm{HR}-20^{\circ} \mathrm{C}\right)$.

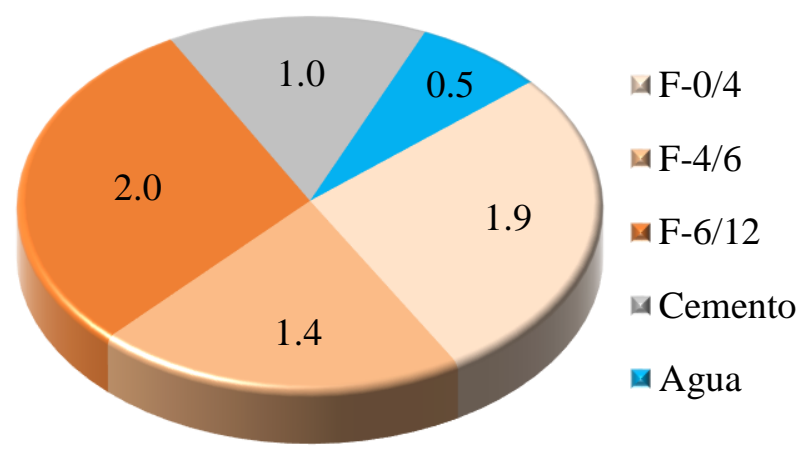

Figura 1. Dosificación de los componentes del hormigón patrón en partes con respecto al cemento. Además del cemento y el agua se detallan las tres fracciones de árido utilizadas.

El cemento utilizado es del tipo CEM I-42,5 R, por tanto con clínker entre un 95-100\% y otros componentes minoritarios entre 0-5\% (RC08, 2008), suministrado por la empresa Holcim en sacos de $25 \mathrm{~kg}$. La ceniza de lodo de depuradora (CLD) procede de la estación depuradora de aguas residualesplanta incineradora de Pinedo (Valencia-España). La ceniza de cáscara de arroz (CCA) suministrada por Maicerías Españolas Dacsa, y fue molida previamente a su uso.

Tabla 1: Dosificación de los hormigones $\left(\mathrm{kg} / \mathrm{m}^{3}\right)$. Las columnas Residuos se refiere a las cantidades de sustitución de cemento por CLD (S-CLD), sustitución de cemento por CCA (S-CCA) y adición de CCA con respecto al cemento (A-CCA)

\begin{tabular}{|c|c|c|c|c|c|c|c|c|}
\hline \multirow{2}{*}{ Referencia muestra } & \multicolumn{3}{|c|}{ Fracciones áridos } & \multirow{2}{*}{ Cem. } & \multicolumn{3}{|c|}{ Residuos } & \multirow{2}{*}{ Agua } \\
\hline & F-0/4 & $F-4 / 6$ & F-6/12 & & S-CLD & S-CCA & A-CCA & \\
\hline $\mathrm{P}$ & 663 & 490 & 714 & 350 & & & & 175.0 \\
\hline 1-A10(CCA) & 663 & 490 & 714 & 350 & & & 35 & 192.5 \\
\hline 2-S(CLD/CCA) & 663 & 490 & 714 & 245 & 70 & 35 & & 175.0 \\
\hline $3-\mathrm{S}(\mathrm{CLD} / \mathrm{CCA})+\mathrm{A} 10(\mathrm{CCA})$ & 663 & 490 & 714 & 245 & 70 & 35 & 35 & 192.5 \\
\hline 4-S(CLD/CCA) & 663 & 490 & 714 & 210 & 70 & 70 & & 175.0 \\
\hline 5-S(CLD/CCA $)+A 10(C C A)$ & 663 & 490 & 714 & 210 & 70 & 70 & 35 & 192.5 \\
\hline
\end{tabular}

Para la fabricación de las probetas han sido utilizados moldes cúbicos de dimensión nominal $150 \mathrm{~mm}$ de arista y el amasado del hormigón se ha llevado a cabo con hormigonera de 50 litros. Las normas de referencia han sido (UNE-EN 12390-1, 2001), (UNE-EN 12390-1/AC, 2001) y (UNE-EN 12390-2.

Los ensayos, realizados en los laboratorios pertenecientes al Dpto. de Ingeniería Civil de la Universidad de Alicante, han sido los siguientes: determinación de velocidad de impulsos ultrasónicos (UNE-EN 12504-4, 2006) y determinación de la resistencia a compresión (UNE-EN 12390-3, 2003) en prensa Suzpecar CMP-150 t. 


\section{RESULTADOS}

En "Tabla 2" y "Figura 2" se muestran los valores medios de las probetas representativas de cada amasada. Lo primero que se podría destacar es que la resistencia a compresión de la mezcla patrón se ve superada por todas las demás, excepto por la mezcla 4, aunque el valor relativo de esta última se aproxima con casi el $96 \%$ del valor de referencia. Pero quizás lo más interesante es que precisamente en la amasada 5, la que más cantidad de residuos contiene, donde mayor Rc se obtiene (34.5 MPa), lo que supone casi un $23 \%$ más que el patrón.

Tabla 2: Resistencia a compresión $(R c)$ y velocidad de impulsos ultrasónicos $(V u)$ en probetas de hormigón. Siendo $\sigma$ la desviación estándar, $C v$ el coeficiente de variación y $R c$ rel y $V u$ rel los valores relativos.

\begin{tabular}{|c|c|c|c|c|c|c|c|c|}
\hline \multirow{3}{*}{ Ref. muestra } & \multicolumn{4}{|c|}{ Resistencia a compresión } & \multicolumn{4}{|c|}{ Velocidad ultrasonidos } \\
\hline & Rc & $\sigma$ & $\mathrm{Cv}$ & $\begin{array}{l}\text { Rc } \\
\text { rel. }\end{array}$ & $\mathbf{V u}$ & $\sigma$ & $\mathrm{Cv}$ & Vu rel. \\
\hline & (MPa) & $(\mathrm{MPa})$ & $(\%)$ & $(\%)$ & $(\mathrm{km} / \mathrm{s})$ & $(\mathrm{km} / \mathrm{s})$ & $(\%)$ & $(\%)$ \\
\hline $\mathrm{P}$ & 28.1 & 1.4 & 4.9 & 100 & 4.61 & 0.05 & 1.1 & 100 \\
\hline 1-A10(CCA) & 30.8 & 2.0 & 6.6 & 109.4 & 4.66 & 0.06 & 1.3 & 101.2 \\
\hline 2-S(CLD/CCA) & 29.7 & 2.1 & 7.0 & 105.6 & 4.63 & 0.06 & 1.4 & 100.4 \\
\hline $3-\mathrm{S}(\mathrm{CLD} / \mathrm{CCA})+\mathrm{A} 10(\mathrm{CCA})$ & 32.5 & 2.0 & 6.3 & 115.5 & 4.65 & 0.08 & 1.8 & 101.0 \\
\hline 4-S(CLD/CCA) & 27.0 & 1.8 & 6.6 & 95.9 & 4.54 & 0.06 & 1.2 & 98.5 \\
\hline $5-\mathrm{S}(\mathrm{CLD} / \mathrm{CCA})+\mathrm{A} 10(\mathrm{CCA})$ & 34.5 & 1.5 & 4.5 & 122.5 & 4.68 & 0.01 & 0.2 & 101.6 \\
\hline
\end{tabular}

Otro dato a destacar es que, si comparamos las mezclas de forma pareada (P-1, 2-3 y 4-5), la adición del 10\% de CCA supone en los tres casos un aumento de la Rc respecto a su homóloga.

Estos aumentos de la Rc al incrementar la cantidad de residuos contenidos en el hormigón, pueden deberse al efecto que producen los residuos tanto como material fino, que completa la distribución granulométrica de los áridos que componen la amasada con un posible aumento de la densidad, como el propio efecto puzolánico de los mismos.

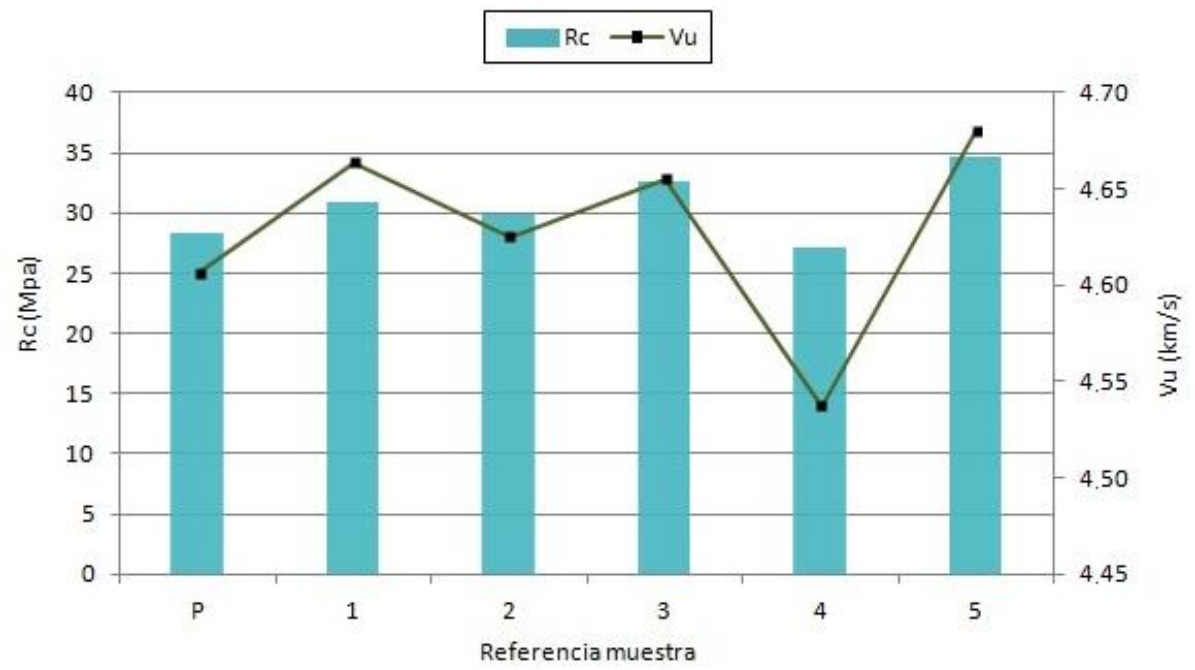

Figura 2: Resistencia a compresión (Rc) y velocidad de impulsos ultrasónicos (Vu) en probetas de hormigón. 
Respecto a la velocidad de los impulsos ultrasónicos se aprecia gráficamente cierta correlación entre ambos parámetros representados en la "Figura 2", lo que se confirma mediante el análisis estadístico y la gráfica de regresión en la "Figura 3": existe una relación estadísticamente significativa entre las variables (Valor-P = 0.019, inferior a 0.05) y moderadamente fuerte entre ellas (Coef. 0.89), de forma que al aumentar la resistencia a compresión, aumenta la velocidad de los impulsos ultrasónicos en los hormigones estudiados.

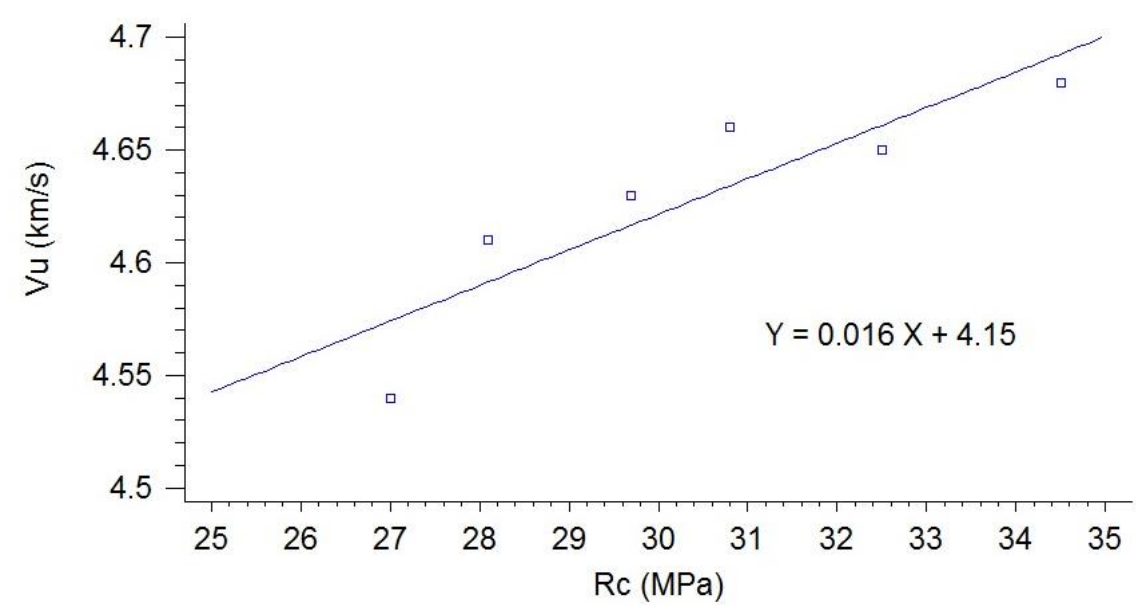

Figura 3: Relación entre resistencia a compresión y velocidad de impulsos ultrasónicos en probetas de hormigón.

\section{CONCLUSIONES}

Los resultados revelan que en los hormigones estudiados con una sustitución de cemento equivalente de hasta el $50 \%$ aproximadamente, la resistencia a compresión supera notablemente la muestra patrón (aproximadamente un 23\%). Los resultados obtenidos podrían deberse al doble efecto de los residuos: por un lado, la matriz es cerrada con el aporte de material fino y, por otro, debe tenerse en cuenta el propio carácter puzolánico de los mismos. Lo anterior aporta indicios de que el uso de manera combinada de CLD y CCA puede ser una alternativa sostenible para mejorar las propiedades mecánicas del material, reduciendo la cantidad de cemento utilizado y valorizando residuos cuyo destino actual suele ser el vertedero.

\section{REFERENCIAS}

Alcocel EG, Garcés P, Martinez JJ, Payá J, Garcia L. (2006), Effect of sewage sludge ash (SSA) on the mechanical performance and corrosion levels of reinforced Portland cement mortars. Mater Construcc. 56:31-43.

Baeza-Brotons, F., Garcés, P., Payá, J., Saval, J.M. (2014a), Portland cement systems with addition of sewage sludge ash. Application in concretes for the manufacture of blocks. Journal of Cleaner Production. 82:112-124.

Baeza-Brotons, F., Payá, Galao, O., Saval, J.M., Garcés, P (2014b), Blending of industrial waste from different sources as partial substitution of Portland cement in pastes and mortars. Construction and Building Materials. 66:645-653 
Baeza Brotons, F., Garcés Terradillos, P., Payá Bernabeu, J., \& Galao Malo, O. (2015). Valorización de cenizas de lodo de depuradora como componente de hormigones para prefabricados. Revista ALCONPAT, 5(1), 44 - 57. doi: http://dx.doi.org/10.21041/ra.v5i1.76

Centro de Estudios y Experimentación de Obras Públicas (CEDEX). Catálogo de Residuos Utilizables en Construcción. [En línea, acceso mayo de 2019. http://www.cedexmateriales.es/].

Chen, M., Blanc, D., Gautier, M., Mehu, J., Gourdon, R. (2013), Environmental and technical assessments of the potential utilization of sewage sludge ashes (SSAs) as secondary raw materials in construction. Waste Manage. 33:1268-75.

Cyr, M., Coutand, M., Clastres, P. (2007), Technological and environmental behaviour of sewage sludge ash (SSA) in cement-based materials. Cem Concr Res. 37:1276-89.

Donatello, S., Tyrer, S., Cheeseman, C.R. (2010), Comparison of test methods to assess pozzolanic activity. Cem Concr Comp. 32:121-7.

Instrucción para la recepción de cementos (RC-08). (2008), [En línea, acceso mayo de 2019 https://www.fomento.gob.es/NR/rdonlyres/49BFE431-DA50-4972-91C6-

DA9EFCB75983/115199/INSTRUC_RECEP_CEMENTOS_consolidada.pdf]

Ley 22/2011, de 28 de julio, de residuos y suelos contaminados (2011), [En línea, acceso mayo de 2019 https://www.boe.es/eli/es/1/2011/07/28/22/con]

Ministerio de Agricultura, Alimentación y Medio Ambiente. Gobierno de España. Plan Estatal Marco de Gestión de Residuos (PEMAR) 2016-2022. (2015), [En línea, acceso mayo de 2019 https://www.miteco.gob.es/es/calidad-y-evaluacion-ambiental/planes-y-

estrategias/pemaraprobado6noviembrecondae_tcm30-170428.pdf].

Monzó, J., Payá, J., Borrachero, M.V., Córcoles, A. (1996), Use of sewage sludge ash (SSA)-cement admixtures in mortars. Cem Concr Res. 26:1389-98.

Payá, J., Monzó, J., Borrachero, M.V., Amahjour, F., Girbé,s I., Velázquez, S., Ordóñez, L.M. (2002), Advantages in the use of fly ashes in cements containing pozzolanic combustion residues: silica fume, sewage sludge ash, spent fluidized bed catalyst and rice husk ash. J Chem Technol Biot. 77:331-5.

Rahmat, M., Ranjbar, M.M., Moghadam, H.A., Mousavi, S.Y. (2011), Mechanical properties and durability assessment of rice husk. Engineering, Biosystems. 144-152.

Rawaid, K. (2012), Reduction in environmental problems using rice-husk ash in concrete. Construction and Building Materials. 30:360-65.

Roskovic, R., Bjegovic, D. (2005), Role of mineral additions in reducing CO2. Cement and Concrete Research. 35 (1):974-978.

UNE-EN 12390-1. (2001), Ensayos de hormigón endurecido. Parte 1: Forma, medidas y otras características de las probetas y moldes.

UNE-EN 12390-1/AC.(2001), Ensayos de hormigón endurecido. Parte 1: Forma, medidas y otras características de las probetas y moldes.

UNE-EN 12390-2. (2001), Ensayos de hormigón endurecido. Parte 2: Fabricación y curado de probetas para ensayos de resistencia.

UNE-EN 12504-4. (2006), Ensayos de hormigón en estructuras.Parte 4: Determinación de la velocidad de los impulsos ultrasónicos.

UNE-EN 12390-3. (2003), Ensayos de hormigón endurecido. Parte 3: Determinación de la resistencia a compresión de probetas. 